\title{
AN OVERVIEW OF COMPUTATIONAL MHD JETS AND THEIR COMPARISONS WITH RECENT HIGH RESOLUTION RADIO IM- AGES
}

\author{
DAVID A. CLARKE \\ National Center for Supercomputing Applications \\ University of Illinois at Urbana-Champaign \\ Champaign, IL 61820, U.S.A.
}

\begin{abstract}
Significant progress has been made in comparing numerical simulations with radio images of astrophysical jets. One is no longer forced to compare density slices through an axisymmetric jet simulation with observed Stokes $I$ images. With the advent of MHD codes and modern supercomputers, models can now be constrained by all four Stokes parameters. In this paper, recent efforts to simulate synchrotron emission images of extragalactic radio jets are reviewed.
\end{abstract}

\section{Introduction}

The fluid model for extragalactic radio sources is well established, and although there are still some questions regarding the basic assumptions of this model (e.g. if the radio plasma is collisional), it is unsurpassed in its ability to explain the structures observed in extragalactic jets and their associated lobes.

Traditionally, density, pressure, kinetic luminosity and various other numerically simulated quantities have been compared directly with the total intensity images obtained from interferometric radio telescopes such as the WSRT, NRAO VLA, MERLIN, VLBI, etc. These comparisons have been very enlightening (see, for example, Norman et al. 1985), but their usefulness has begun to wane, primarily for three reasons. First, they can constrain a model for extragalactic radio sources with just one Stokes parameter (Stokes $I$ ). Second, the Stokes $I$ parameter integrated along the line of sight and a density slice taken along the symmetry axis of a 2$\mathrm{D}$ simulation are very different quantities. Third, all of the high resolution jet simulations reported to date are restricted to two spatial dimensions.

The generalisation of hydrodynamical (HD) numerical codes to magnetohydrodynamical (MHD) codes addresses the first two of these three limitations, and has given rise to the notion of "numerical observations". With a self-consistent computation of the magnetic field, the Stokes parameters integrated along the line of sight can be computed for a simulation of a radio jet and its associated lobe (Matthews 1989; Clarke, Norman, and Burns 1989, hereafter CNB). From these quantities, the total synchrotron intensity, fractional polarisation, and polarisation position angle can be computed and compared directly with those obtained from the radio interferometers. This advance is the primary focus of this paper. 


\section{Early Work}

Laing (1981) analytically computed the synchrotron appearance of a uniform, static, cylindrical jet with various magnetic field configurations, both ordered and random. By appending a hemispherical cap to the end of the cylinder, Laing reproduced many of the gross structures seen in the polarisation data from extragalactic radio jets. These include the general trend of the polarisation $\mathbf{E}$-vectors to be circumferentially perpendicular and axially parallel, and for the fractional polarisation to be the highest along the source boundary. He pointed out that the combination of geometrical effects, such as the orientation of the jet, and physical effects, such as the magnetic field configuration, prohibited a unique inference of the true magnetic field in the source from the polarisation data.

The first attempt to compute the synchrotron emission from a numerically generated, axisymmetric jet was by Smith et al. (1985). Since their code did not incorporate magnetic fields, they used equipartition arguments to obtain an emissivity as a function only of the thermal pressure. Then, by rotating the emissivity about the symmetry axis, they were able to integrate the Stokes $I$ parameter along the line of sight through the solid of revolution at various orientations of the jet axis. They were primarily interested in the structures of the leading hotspot.

Smith et al. found that the emission patterns depend strongly on orientation of the jet, and epoch chosen. Figure 1 shows some of the results from their calculation of a jet with a Mach number $(M)$ of 6 and a density ratio $(\eta)$ of 0.1 (compared with the ambient medium) at two different epochs and two different orientations. It is clear that no unique hotspot morphology exists for a given value of $M$ and $\eta$. Additionally, no unique values for $M$ and $\eta$ exist given a single hotspot. Thus, the jet parameters cannot be determined from the hotspot alone.

Nevertheless, Smith et al. classify the various hotspot morphologies found in their simulations, and note that most of these can be identified in nature. This study helped to provide the foundation for the more recent MHD simulations, from which all four Stokes parameters can, in principle, be computed.

\section{MHD Jet Calculations}

Although there are many groups within the astrophysical, physics, engineering, and military communities developing sophisticated MHD codes, only a handful are being applied to astrophysical jets, and fewer still where the synchrotron appearance of the simulation is being specifically computed. It is the results of these studies that will be discussed here.

Matthews (1989) evolved an axisymmetric jet $(M=10, \eta=0.1)$ for almost two sound crossing times (jet radius divided by the sound speed of the unperturbed ambient medium). A poloidal magnetic field (in the plane of the calculation) was represented by a set of unit vectors with a random initial orientation which were advected with the jet material. No toroidal component was considered, and the magnetic field was assumed to be dynamically negligible (i.e. passive, $\beta \equiv 2 \mu_{0} p / B^{2} \gg 1$ ). Synchrotron aging of the relativistic electrons was incorporated as a function of the magnetic field strength while reacceleration processes were ignored. Figure 2 shows the synchrotron appearance of their jet viewed at an angle of $75^{\circ}$ between the jet axis and the line of sight for four different RMS magnetic field strengths.

Despite the randomness of the initial magnetic field, they find that the synchrotron emission is significantly polarised, sometimes as high as the theoretical 


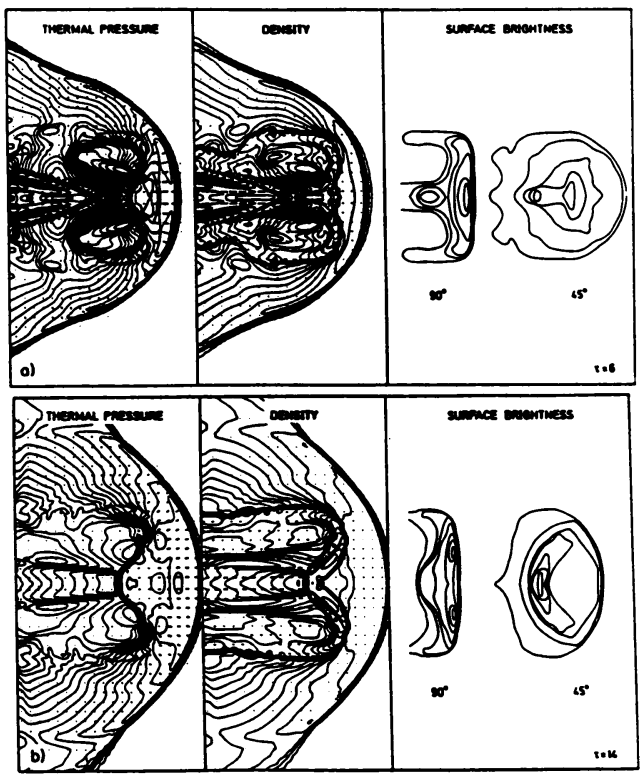

FIG URE 1. The terminal hotspots of a computed jet at two different epochs. Note the different shock structures (pressure) and contact discontinuity shapes (density) which result in the radically different hotspot morphologies (Smith et al. 1985).
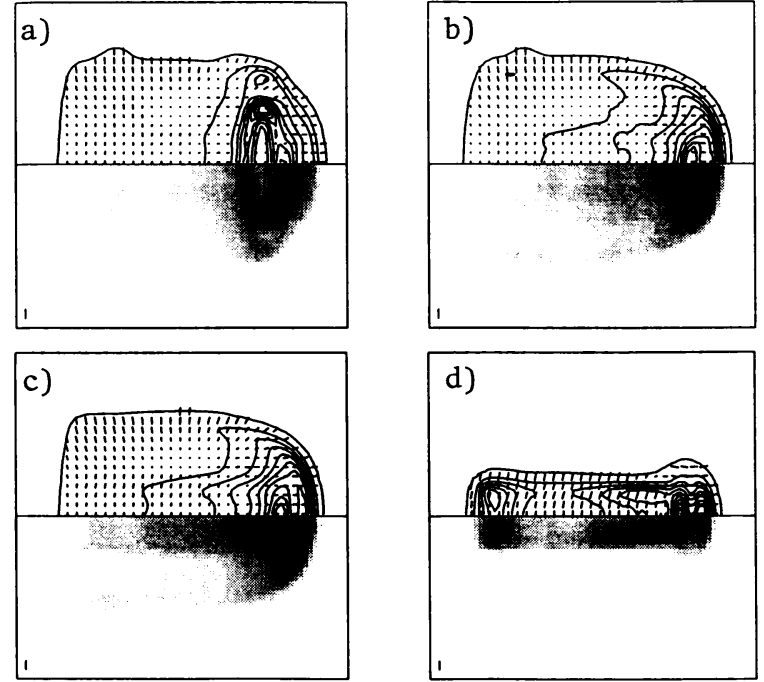

FIG URE 2. Total intensity contours, with polarisation E-vectors superposed (top half) and total intensity grey scale (bottom half) for a simulated jet with four RMS magnetic field strengths: a) $0 \mu G, b) 1 \mu G, c) 10 \mu G, d) 100 \mu G$ (Matthews 1989). 
limit. Thus, shear and shock compression partially order the magnetic field. In the absence of synchrotron aging (Figure $2 a$ ), the dominant emission feature is a top- and bottom-brightened ring at the head of the jet. This results when a small region, whose magnetic field is grossly amplified by vortically induced shear, is spun about the symmetry axis. Thus, it is partly an artefact of the imposed geometry. Because of its relatively high magnetic field strength, the feature disappears when synchrotron aging is accounted for (Figures $2 b, 2 c$, and $2 d$ ), yielding to a centrefilled terminal hotspot. Note the $90^{\circ}$ flip in direction of the E-vectors near the head of the jet. This is a natural consequence of the flow, in which a predominantly axial magnetic field is deflected radially by the ram pressure of the ambient medium.

CNB evolved an axisymmetric jet $(M=6, \eta=0.1)$ for 16 sound crossing times. A passive, helical magnetic field was specified at the jet orifice, and allowed to advect with the flow. In this case, the hydrodynamics of the flow randomises the magnetic field, rather than ordering it, as seen in Matthews (1989). Despite this difference, the results of the two simulations are qualitatively similar. When determining the synchrotron appearance of the jet, CNB vary the initial pitch angle of the magnetic field $\left(\gamma \equiv \tan ^{-1}\left(B_{\phi} / B_{z}\right)\right)$ and the angle between the symmetry axis and the line of sight $(\delta)$. Figure 3 illustrates the synchrotron appearance of the jet at the final epoch of the simulation for $\gamma=30^{\circ}$, and four values of $\delta$.

The synchrotron appearance of the jet depends strongly on the parameters $\gamma$ and $\delta$, and the epoch of the calculation observed. Of course, the Mach number, density ratio, and profile of the ambient medium play a role too. However, the enormity of this parameter space prohibits an exhaustive parameter survey, and thus, CNB draw the following broad generalisations from this single calculation.

1. A jet with only a passive toroidal component of the magnetic field is virtually obscured by the surrounding cocoon. For smaller pitch angles, the jet is increasingly bright relative to the background emission. This stems from the different rates at which the toroidal and poloidal magnetic field components diminish away from the symmetry axis $\left(r^{-1}\right.$ vs. $\left.r^{-2}\right)$. Thus, a cocoon with a predominantly toroidal magnetic field will contribute more to the line-of-sight emission than the jet.

2. The brightness along the jet is far from uniform. The synchrotron intensity is enhanced by the highly oblique, "criss-cross" shocks, which compress the axial magnetic field component. At the epoch shown, these shocks are found only along the first half of the jet, corresponding to the sudden drop in synchrotron emissivity. The polarisation E-vectors flip from transverse to longitudinal relative to the jet axis, right where the jet disappears (e.g. the jet in $3 \mathrm{C} \mathrm{219}$, Figure 4).

3. A top- and bottom-darkened emission ring ("hamburger hotspot") dominates the total intensity at the head of the jet for moderate values of $\delta$ (Figures $3 b$ and $3 c)$. Unlike the ring reported by Matthews (1989, Figure $2 a)$, this ring is a result of obliquely viewing a ram-compressed, passive toroidal magnetic field component (e.g. the hotpsot in the western lobe of Cygnus A; Carilli et al. 1989).

4. For small $\delta$, an elliptical pattern of radially pointing polarisation $\mathbf{E}$-vectors is seen at the head of the jet. Accompanying this pattern are two "warm spots" in the total intensity, which are remnants of the "hamburger hotspot" when the jet is

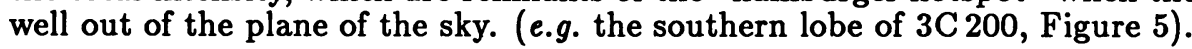

5. Along the circumference of the emission, the polarisation E-vectors are perpendicular, for almost all combinations of $\delta$ and $\gamma$, consistent with the findings of Laing (1981), Matthews (1989), and the vast majority of the radio observations. 

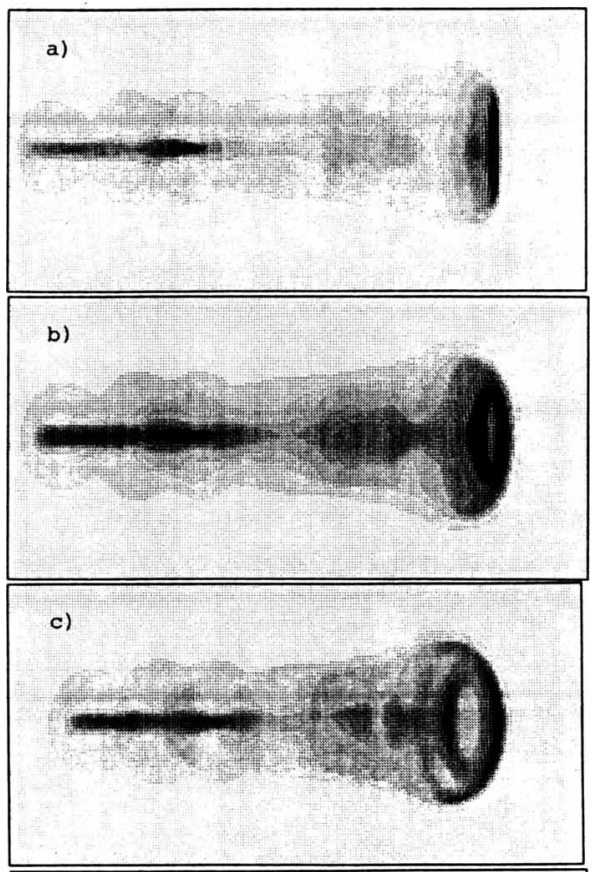

d)

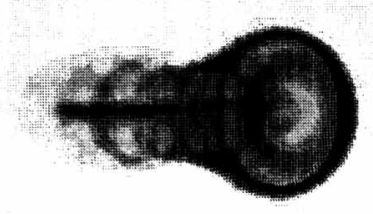

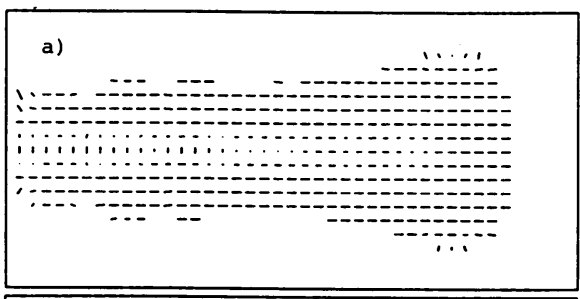
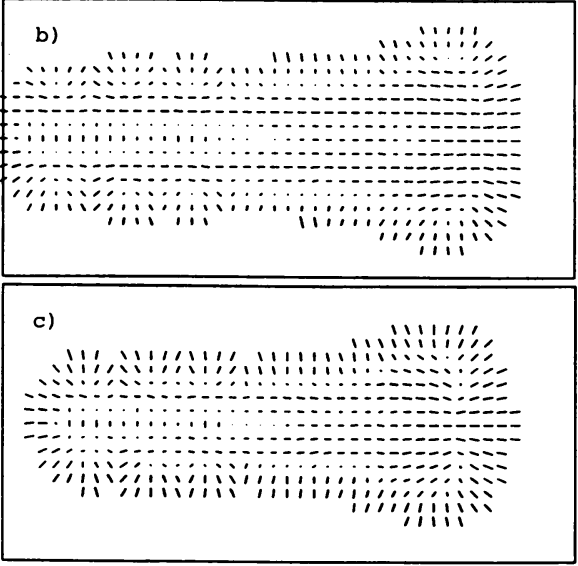

d)

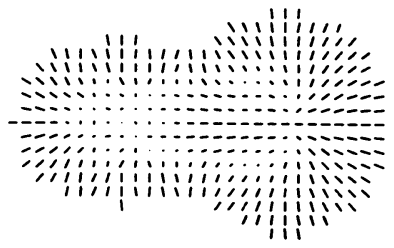

FIgURE 3. Total intensity grey scale (left) and polarisation E-vectors (right) of a simulated jet with an initially helical magnetic field $\left(\gamma=30^{\circ}\right)$ for four viewing angles: a) $90^{\circ}$ (in the plane of the sky), b) $75^{\circ}$, c) $60^{\circ}, d$ ) $30^{\circ}$ (CNB). The images are convolved with a circular beam (FWHM $\sim 0.7 r_{\text {jet }}$ ).

Turning now to an active magnetic field $(\beta \lesssim 1)$, the parameter space becomes even larger, and general conclusions based on a small number of simulations are elusive. Nevertheless, some preliminary findings do exist. Clarke et al. (1986) present the first calculation of a magnetically confined jet (Figure 6). The most important physical difference between this and the HD jet is the fate of the material thermalised by the terminal Mach disc. In an HD jet with a low $\eta$, this material is deflected by the ram pressure of the ambient medium and inflates the cocoon. In the magnetically confined jet, this material is confined at the head of the jet by the $\mathbf{J} \times \mathbf{B}$ forces, forming a "nose-cone". The transonic "nose-cone" can be as long as the supersonic portion of the jet and separates the bow shock from the terminal Mach disc. Additional MHD effects can be seen along the jet itself. The "crisscross" shocks are stronger than in the HD jet and less oblique. The cocoon, which is not inflated by processed jet material, is narrow, rarefied, and highly magnetised. 


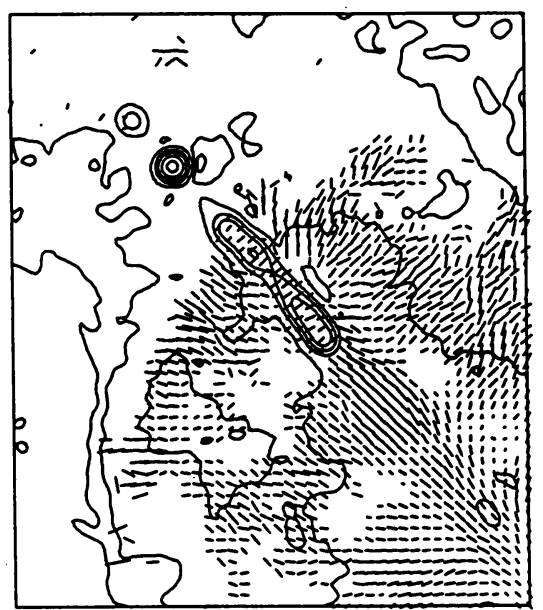

FIG URE 4. VLA total intensity contour image (22 cm, 1".4 resolution) of the jet in $3 \mathrm{C} 219$, with polarisation E-vectors superposed. Note the $90^{\circ}$ flip in the E-vectors right where the jet seems to end.

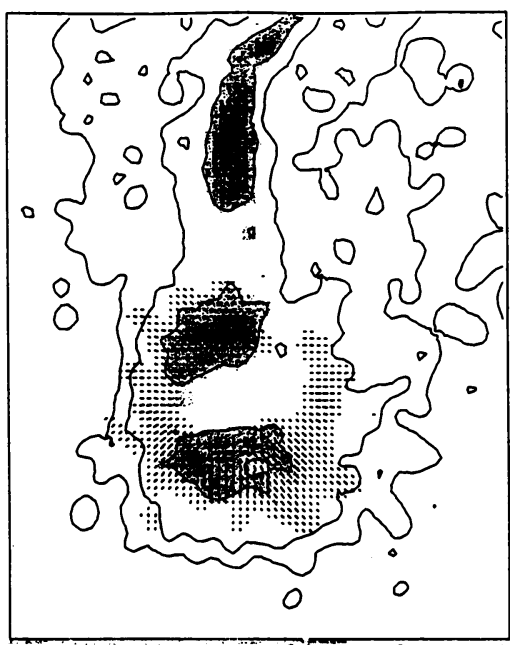

FIG URE 5. VLA total intensity contour and grey scale image $(6 \mathrm{~cm}, 0$ !'3 resolution) of the southern lobe of $3 \mathrm{C} 200$ with the polarisation E-vectors superposed.

The synchrotron appearance of this jet (Figure 7) is knotty, naked, and dominated by the bright, elongated "nose-cone". The transonic nature of the "nose-cone" substantially reduces the importance of shock reacceleration of relativistic electrons. Thus, a spectral steepening along the length of the feature might be expected. Note that along the jet axis, the E-vectors are parallel, indicative that only a toroidal magnetic field is considered in this calculation. If the magnetic field had a significant axial component, the $\mathbf{E}$-vectors could be transverse on axis, such as those seen in Figure 3 and in better agreement with observations of the powerful radio sources. However, a jet with an active helical magnetic field may behave very differently from a jet in which the active magnetic field is purely toroidal (Kössl 1989).



FIG URE 6. Density contours for the magnetically confined jet $(M=6, \eta=0.1$, $\beta=0.4$, Clarke et al. 1986). 
Examples of a magnetically confined jet may include 3C 273 (Davis et al. 1985, Figure 8 ) and 0800+608 (Shone and Browne 1986, Figure 9). Both jets are clumpy, naked, and possess an elongated, bright feature at their leading ends. There is also evidence of spectral aging along the "nose-cone" of 3C 273 (Röser and Meisenheimer $1986 ;$ R. A. Perley 1988, private communication). However, the total intensity along
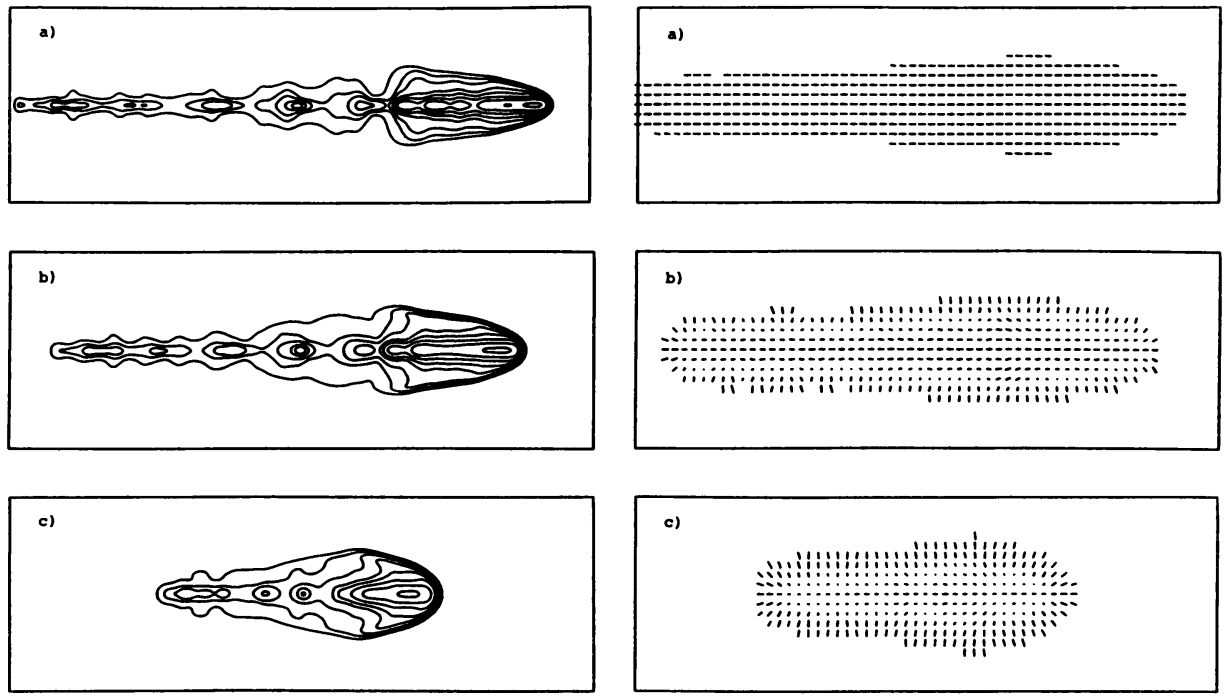

FIG URE 7. Total intensity contours (left) and polarisation E-vectors (right) for the magnetically confined jet in Figure 6 for three viewing angles: $\left.a) 90^{\circ}, b\right) 60^{\circ}, c$ ) $30^{\circ}$. The images are convolved with a circular beam (FWHM $\sim 0.5 r_{\text {jet }}$ ).

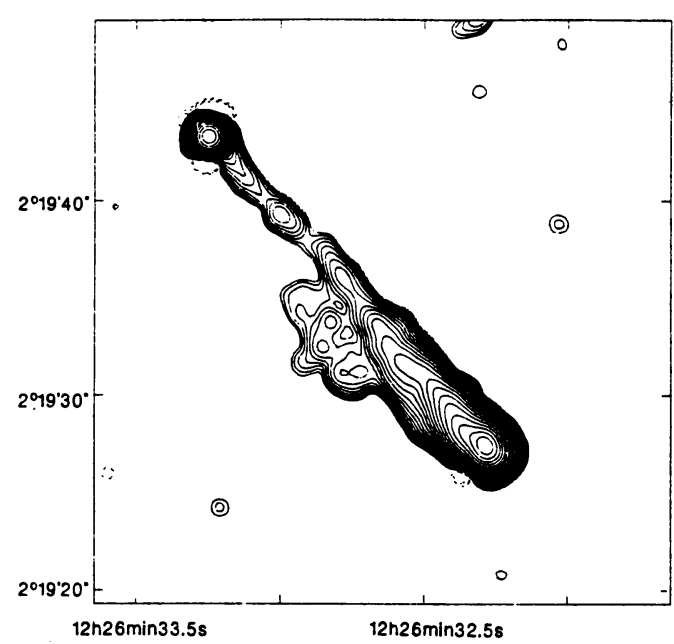

FIG URE 8. MERLIN image $\left(75 \mathrm{~cm}, 1^{\prime \prime}\right.$ resolution) of the jet in $3 \mathrm{C} 273$ (Davis et al. 1985).

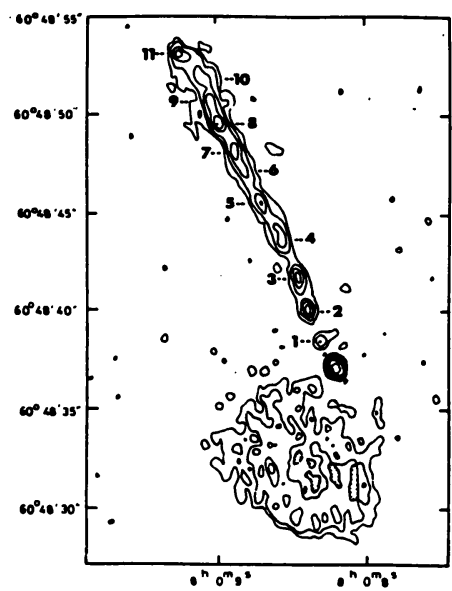

FIG URE 9. VLA image $(6 \mathrm{~cm}, 0.5$ resolution) of the radio source $0800+608$ (Shone and Browne 1986). 
the "nose-cone" of $3 \mathrm{C} 273$ rises exponentially, a behaviour not observed along the "nose-cone" of the simulated jet (R. G. Conway 1989, private communication).

\section{Final Remarks}

With the development of MHD codes, modeling of extragalactic radio jets is now constrained by three observables, namely the total intensity, polarised intensity, and polarisation position angle. Various observable signatures to distinguish between geometrical effects (e.g. line-of-sight angle) and physical effects (e.g. magnetic field configuration) have emerged. The very existence of an extended cocoon and many of the observed polarisation patterns may be a consequence of a passive magnetic field. Thus, it may be that the magnetic field permeating the radio plasma of most extragalactic radio sources is, on average, dynamically weak.

Yet, there are still many unanswered questions. Some results of the numerical simulations may be exaggerated by the imposed symmetry and can only be verified by fully 3-D calculations. With the availability of the Cray-2 supercomputer, results from high resolution, 3-D simulations will soon be available. The relativistic particles should be treated as a separate component in the jet to account for the aging and reacceleration of the synchrotron electrons. Thus, spectral index images of jets will be simulated, placing even stronger constraints on the models.

I thank my collaborators Michael Norman and Jack Burns for their invaluable contributions to some of the work reviewed here. I also thank M. Smith, A. Matthews, R. Davis, and D. Shone for permission to reproduce Figures 1, 2, 8, and 9 respectively. Travel support from the Deutsche Forschungsgemeinschaft and the hospitality of the Institute for Astrophysics at UNM are gratefully acknowledged. Some of the calculations reviewed here were supported by NSF grant AST-87003 to the National Center for Supercomputing Applications, University of Illinois.

\section{References}

Carilli, C. L., Dreher, J. W., and Perley, R. A. 1989, in Hot Spots in Extragalactic Radio Sources, Proceedings of the Ringberg Castle Workshop, editors K. Meisenheimer and H.-J. Röser, (Berlin: Springer-Verlag), 51.

Clarke, D. A., Norman, M. L., and Burns, J. O. 1986, Ap. J. (Letters), 311, L63. . 1989, Ap. J., 342, 700 (CNB).

Davis, R. J., Muxlow, T. W. B., and Conway, R. G. 1985, Nature, 318, 343.

Kössl, D. 1989, in Hot Spots in Extragalactic Radio Sources, Proceedings of the Ringberg Castle Workshop, editors K. Meisenheimer and H.-J. Röser, (Berlin: Springer-Verlag), 225.

Laing, R. A. 1981, Ap. J., 248, 87.

Matthews, A. P. 1989, in Hot Spots in Extragalactic Radio Sources, Proceedings of the Ringberg Castle Workshop, editors K. Meisenheimer and H.-J. Röser, (Berlin: Springer-Verlag), 219.

Norman, M. L., Smarr, L. L., Winkler, K.-H. A. 1985, in Numerical Astrophysics, ed. J. Centrella, J. LeBlanc and R. Bowers (Boston: Jones and Bartlett), 88.

Röser, H.-J., and Meisenheimer, K. 1986, Astron. Astrophys., 154, 15.

Shone, D., and Browne, I. W. A. 1986, M.N.R.A.S., 222, 365.

Smith, M. D., Norman, M. L., Winkler, K.-H. A., and Smarr, L. 1985, M.N.R.A.S., 214, 67. 
PERATT: Relativistic jets, or intense relativistic electron beams, have been a topic of considerable interest outside the astronomical community for the last 5 or 6 years. This derives from initial claims that such a jet can propagate, collimated, for distances of $\mathrm{kpc}$ or hundreds of $\mathrm{kpc}$. In the laboratory one encounters instabilities and filamentation in beam propagation experiments. Since this behavior is not apparent in astrophysical "jets", are you sure their interpretation as outflowing matter is correct?

CLARKE: It is not true that instabilities and filamentation are not observed in astrophysical jets. In the jets of Centaurus A and M87, which are the closest such objects to the Earth, filamentation is very apparent. Although jets in other radio sources are too far away to be resolved enough to see the filamentation, kink instabilities are quite common and is widely believed to be a primary reason why jets break up to form their lobes. In the laboratory, technical problems make simulating jets with parameters analogous to astrophysical jets (e.g. density ratio, Mach number, plasma beta) very difficult, so comparing laboratory jets with astrophysical jets may not be appropriate. Numerically, simulations show that astrophysical jets are unstable, but not so unstable as to prevent them from being conduits of matter from the core to the radio lobe.

CONWAY: It is observed in high-luminosity (FR II) sources with 2 lobes that only one jet is detected, the ratio in emission typically at least 10-100. This is so common that it cannot be due to special cases or a contrived geometry. Is there anything in your simulation which could lead to such an asymmetry?

CLARKE: In order to apply these simulations to the overall question of sidedness in Class II radio sources, one needs to devise a mechanism which would predispose the nucleus of the galaxy to launch one jet with a helical magnetic field of moderate pitch angle (leading to the detected main jet) and the other jet with a toroidal field (leading to the undetected counterjet). As of this time, I have no such mechanism in mind.

SCHEUER: There is no reason why a randomly tangled input field (as in Alan Matthews' work) should not be used in the case where the magnetic pressure tensor becomes dynamically important.

Matthews and I found that, even starting with a totally disordered field, we got polarizations close to the theoretical maximum over large parts of the simulated source - polarizations much higher than those typical in observations. We conclude that a great deal of field line reconnection must occur. Do you also get such high polarizations? If there is indeed much reconnection, that will profoundly change the dynamical effects of the magnetic field in any simulation.

CLARKE: Yes, we do see large regions of high polairzation ( $>50 \%$ ) in our simulated emission images as well. These areas are typically restricted to the jet and to the outermost regions of the cocoon. In the jet, the supersonic flow is not turbulent, and the ordered field remains as such giving rise to the high degree of polarization. In the outermost regions of the cocoon, the high degree of polarization is undoubtedly caused by inte- 
grating through only a few zones, resulting in a minimal degree of field crossing. Reconnection events, through small-scale turbulent eddies, will certainly reduce the degree of polarization, but I do not agree that this will necessarily affect the dynamics of the jet. In particular, when the field is passive, such as in your simulations, the details of the magnetic field structure do not affect the evolution of the system at all.

KUNDT: At this meeting, jet material has been assumed to consist of hydrogen and to move non-relativistically, despite the many indications of their consisting of pair plasma moving relativistically at a high (bulk) Lorentz factor (such as their superluminal expansion, sidedness, hard spectra, and a peak of the core power near MeV energy; see NATO ASI C 208, W. Kundt (ed.), Reidel, 1987). In any case, particle densities $\mathrm{n}=\mathrm{L} / \mathrm{AEv}$ ( $\mathrm{L}=$ power, $\mathrm{A}=$ area, $\mathrm{E}=$ particle energy, $\mathbf{v}=$ bulk velocity) are so low that an MHD treatment with a pressure-gradient term would have to be justified.

CLARKE: The collisional nature of the radio plasma is still very controversial. Yet, the fluid model has been very successful in explaining the basic structures that we see in the radio sources. Perhaps this can be taken as a posteriori evidence that the radio plasma is somehow collisional in nature. On the other hand, a theory to supplant the fluid model could conceivably be devised. One should keep in mind, however, that collisions of particles with Alfvén waves, which have significantly higher cross sections than particle-particle collisions, could make the radio plasma collisional, validating an MHD treatment.

PERATT: The term "jet" was first used in astrophysics by Curtis in 1918 to describe the elongated optical feature protruding from the core of the elliptical galaxy M87. He used this term because the feature "looked" like a jet. However, independent measurements over the period 1934-1982 suggest that the "jet" is variable and has been fading more or less uniformly by about $0.8 \mathrm{mag}$ per decade between 1964 and 1980 . The data imply that over the period 1952-1980, the total jet intensity fell by at least $2.5 \mathrm{mag}$. Comparisons of isophotes taken in 1964 and 1979 show no obvious differences in overall shape.

At a distance of $11.4 \mathrm{Mpc}$, the channel length of $\mathrm{M} 87$ is 30 arcsec in the plane of the sky, or 5400 light years across. Unless the data can be discounted, or an explanation given as to why a 5400 light year long jet should uniformly fade in 16 years, the interpretation of "jets" as outflowing matter is questionable.

MOUSCHOVIAS (concerning a computer video by Burns): I'm not sure I saw the following correctly, but it looked like the shock surface near the left boundary oscillated. Is it a physical effect or the result of boundary conditions? If the oscillation is real, what do you think causes it?

BURNS: The external shock is not static but moves about in response to the changes in structure of the expanded jet produced by large-scale turbulence. We were careful to ensure that the boundary of the grid did not artificially influence the jet or external medium flows. 